\title{
Applications of combinatorial groups to Hopf invariant and the exponent problem
}

\author{
JELENA GRBIĆ
}

JIE WU

\begin{abstract}
Combinatorial groups together with the groups of natural coalgebra transformations of tensor algebras are linked to the groups of homotopy classes of maps from the James construction to a loop space. This connection gives rise to applications to homotopy theory. The Hopf invariants of the Whitehead products are studied and a rate of exponent growth for the strong version of the Barratt Conjecture is given.
\end{abstract}

55P35; 55Q25, 55Q15, 16W30

\section{Introduction}

The study of the groups of homotopy classes of maps from one topological space to another has always been the central problem of algebraic topology. In this paper we are concerned with the natural maps from loop suspensions to loop spaces. To study them we develop a method which arises from the relations between combinatorial groups and the natural coalgebra transformations of tensor algebras established in the predecessor [6] to this paper. In particular, let $R$ be a commutative ring with identity, and let $\operatorname{Coalg}(A(-), B(-))$ denote the group of natural coalgebra transformations over $R$ between functors $A$ and $B$. The tensor algebra $T(V)$ generated by a free $R$-module $V$ has a natural coalgebra filtration (the James filtration) $\left\{J_{n}(V)\right\}_{n \geq 0}$ given by $J_{n}(V)=\bigoplus_{j \leq n} T_{j}(V)$ for $n \geq 0$, where $T_{j}(V)$ denotes the $j$-th stage of the tensor length filtration for $T(V)$, that is, $T_{j}(V)=V^{\otimes j}$. Let $C(V)=J_{1}(V)$. For two $R$-modules $C$ and $D$, define their smash product $C \wedge D$ to be the quotient module

$$
C \wedge D=(C \otimes D) /\left(C \otimes_{R} R \oplus R \otimes_{R} D\right) .
$$

By $V^{\wedge n}$ we denote the $n$-fold self smash product of $V$.

In [6] we defined combinatorial groups $K_{n}^{R}, \mathcal{H}_{n}^{R},{ }^{R} \mathcal{H}_{n}^{(l)},{ }^{R} \mathcal{H}_{n}^{(l),(k)}$ and $K_{n}^{R}(k)$ such that there are group isomorphisms:

$$
\begin{array}{ll}
\left.\operatorname{Coalg}\left(C(-)^{\otimes n}\right), T(-)\right) \cong K_{n}^{R} & \operatorname{Coalg}\left(J_{n}(-), T(-)\right) \cong \mathcal{H}_{n}^{R} \\
\operatorname{Coalg}\left(J_{n}\left(-{ }^{\otimes l}\right), T(-)\right) \cong \mathcal{H}_{n}^{(l)} & \operatorname{Coalg}\left(J_{n}\left({ }^{\otimes l}\right), T\left(-{ }^{\otimes k}\right)\right) \cong{ }^{R} \mathcal{H}_{n}^{(l),(k)} \\
\left.\operatorname{Coalg}\left(C(-)^{\otimes n}\right), T\left(-^{\wedge k}\right)\right) \cong K_{n}^{R}(k) &
\end{array}
$$


for $1 \leq n \leq \infty$. As a consequence of these group identifications and the fact that Coalg $(A(-), B(-))$ is contained inside the algebra of natural linear transformations $\operatorname{Hom}_{R}(A(-), B(-))$, there exist faithful representations of the above combinatorial groups in the algebras of natural linear transformations of tensor algebras.

The first issue we address in this paper is a geometrical realization of the stated algebraic relations. Restrict the commutative ring $R$ to $\mathbb{Z}$ or $\mathbb{Z} / p^{r}$. Let $X$ be a space such that its reduced diagonal $\bar{\Delta}: X \longrightarrow X \wedge X$ is null homotopic. Denote the $n$-fold self smash product of $X$ by $X^{(n)}$. The connection between the combinatorial groups $K_{n}^{R}$, $\mathcal{H}_{n}^{R},{ }^{R} \mathcal{H}_{n}^{(l)},{ }^{R} \mathcal{H}_{n}^{(l)(k)}$ and $K_{n}^{R}(k)$ and the groups of homotopy classes of maps from the James construction on spaces with a null homotopic reduced diagonal to a loop space will be given by constructing injective group homomorphisms:

$$
\begin{array}{ll}
e_{X}: K_{n}^{R} \longrightarrow\left[X^{n}, J(X)\right] & e_{X}: \mathcal{H}_{n}^{R} \longrightarrow\left[J_{n}(X), J(X)\right] \\
e_{X}:{ }^{R} \mathcal{H}_{n}^{(l)} \longrightarrow\left[J_{n}\left(X^{(l)}\right), J(X)\right] & e_{X}:{ }^{R} \mathcal{H}_{n}^{(l)(k)} \longrightarrow\left[J_{n}\left(X^{(l)}\right), J\left(X^{(k)}\right)\right] \\
e_{X}: K_{n}^{R}(k) \longrightarrow\left[X^{n}, J\left(X^{(k)}\right)\right] &
\end{array}
$$

(see Propositions 2.3, 2.8, 2.13, 2.19, 2.24).

We then proceed to apply the just established group-geometrical model to problems in homotopy theory. In 1931 Hopf defined what is nowadays known as the Hopf invariant in order to study maps between spheres of different dimensions which cannot be distinguished homologically. Ever since, Hopf invariants and their relations with Whitehead products have been widely studied (see for instance Cohen and Taylor [5] and $\mathrm{Wu}[12]$ ), and these have various applications in homotopy theory to the homotopy groups of spheres, exponent problems and LS-category. The first application of our group-geometrical model is to calculate the generalized $k$-th Hopf invariant of the $n$-fold Whitehead product when $k$ does not divide $n$ and to determine the second Hopf invariant of the 4 -fold Whitehead product.

Recall that for any pointed space $X$, the James-Hopf map $H_{k}: J(X) \longrightarrow J\left(X^{(k)}\right)$ is combinatorially defined by

$$
H_{k}\left(x_{1} x_{2} \ldots x_{n}\right)=\prod_{1 \leq i_{1}<i_{2}<\cdots<i_{k} \leq n}\left(x_{i_{1}} x_{i_{2}} \ldots x_{i_{k}}\right)
$$

with right lexicographical order in the product. The $n$-fold Samelson product $\widetilde{W}_{n}$ on $X$ is given by the composite

$$
\widetilde{W}_{n}: X \wedge \ldots \wedge X \stackrel{E \wedge \ldots \wedge E}{\longrightarrow} \Omega \Sigma X \wedge \ldots \wedge \Omega \Sigma X \stackrel{[[,], \ldots,]}{\longrightarrow} \Omega \Sigma X
$$


where $E: X \longrightarrow \Omega \Sigma X$ is the canonical inclusion and the second map $[[],, \ldots$,$] is$ the $n$-fold commutator. The $n$-fold Whitehead product $W_{n}$ on $X$ is defined as the adjoint of the $n$-fold Samelson product $\widetilde{W}_{n}$.

Theorem 1.1 Let $X$ be a pointed space with the null homotopic reduced diagonal $\bar{\Delta}: X \longrightarrow X \wedge X$. Then for $n>k$,

$$
J\left(X^{(n)}\right) \stackrel{\Omega W_{n}}{\longrightarrow} J(X) \stackrel{H_{k}}{\longrightarrow} J\left(X^{(k)}\right)
$$

is null homotopic if $k$ does not divide $n$.

The case when $k$ divides $n$ is much more subtle. Here we determine the first non-trivial case, that is, the second Hopf invariant of the 4-fold Whitehead product, leaving the description of the general case as a future project.

Theorem 1.2 For $X$ as in Theorem 1.1, define the map $\Phi: \Sigma X^{(4)} \longrightarrow \Sigma X^{(4)}$ as

$$
\Phi=\tau_{34}-\tau_{12} \tau_{34}+\tau_{14} \tau_{23}+\tau_{13} \tau_{24}
$$

where $\tau$ is the twist map. Then there is a commutative diagram

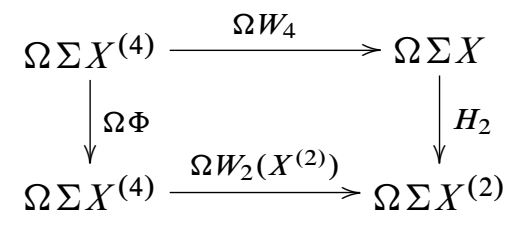

where $W_{n}$ denotes the $n$-th fold Whitehead product and $H_{2}$ the second James-Hopf map.

The second application of our method is concerned with exponent problems in homotopy theory. In general, two types of exponents of a given space $X$ can be considered. Let $p$ be a prime. The mod $p$ homotopy exponent of a space $X$ is $p^{r}$ if that is the least power of $p$ which annihilates the $p$-torsion component of $\pi_{*}(X)$. Denote the mod $p$ homotopy exponent of $X$ by $\exp (X)=p^{r}$. A stronger notion is that of a multiplicative (or $H$-) exponent. If $Y$ is an $H$-space, then the $p$-th power map is given by the composite $p: Y \stackrel{\Delta}{\longrightarrow} Y \otimes p \stackrel{\mu}{\longrightarrow} Y$, where $\mu$ is the multiplication on $Y$. The multiplicative exponent of $Y$ is $p^{r}$ if that is the least power of $p$ such that $p^{r}: Y \longrightarrow Y$ is null homotopic, while $p^{r-1}: Y \longrightarrow Y$ is essential. We say that $Y$ has no multiplicative exponent if the $p^{r}$-th power map on $Y$ is essential for all $r \in \mathbb{N}$. A major exponent conjecture was posed by Barratt. 
The Barratt Conjecture Let $f: \Sigma^{2} X \longrightarrow Y$ be a map of order $p^{r}$ in $\left[\Sigma^{2} X, Y\right]$. Then

$$
p^{r+1} \operatorname{Im}\left(f_{*}: \pi_{*}\left(\Sigma^{2} X\right) \longrightarrow \pi_{*}(Y)\right)=0 .
$$

In particular, if the identity map on $\Sigma^{2} X$ is of order $p^{r}$ in $\left[\Sigma^{2} X, \Sigma^{2} X\right]$, then the mod $p$ exponent of $\Sigma^{2} X$ is $p^{r+1}$, that is,

$$
p^{r+1} \pi_{*}\left(\Sigma^{2} X\right)=0 .
$$

A strong version of the Barratt Conjecture is concerned with the multiplicative exponent of $\Sigma^{2} X$ and can be stated as follows.

The Strong Barratt Conjecture Let $f: \Sigma^{2} X \longrightarrow Y$ be a map of order $p^{r}$ in $\left[\Sigma^{2} X, Y\right]$. Then

$$
\Omega^{2} f: \Omega^{2} \Sigma^{2} X \longrightarrow \Omega^{2} Y
$$

has order bounded by $p^{r+1}$ in $\left[\Omega^{2} \Sigma^{2} X, \Omega^{2} Y\right]$.

We start with a map $f: X \longrightarrow \Omega Y$ such that $p^{r}[f]=0$ in the group $[X, \Omega Y]$. Let $J(f): J(X) \longrightarrow \Omega Y$ be its multiplicative extension to the James construction. Using the combinatorial description of the group of homotopy classes of maps from the James filtration $\left\{J_{n}(X)\right\}_{n \geq 0}$ to a loop space, we estimate the rate of growth of the order of the map $J(f)$.

Theorem 1.3 Let $X=\Sigma X^{\prime}$ be a suspension and let $f: X \longrightarrow \Omega Y$ be a map such that $p^{r}[f]=0$ in the group $[X, \Omega Y]$. Let $J(f): J(X) \longrightarrow \Omega Y$ be the canonical multiplicative extension of $f$. Then the following hold.

(1) The map

$$
\left.J(f)\right|_{J_{n}(X)}: J_{n}(X) \longrightarrow \Omega Y
$$

has order $p^{r+t}$ in $\left[J_{n}(X), \Omega Y\right]$ if $n<p^{t+1}$.

(2) The composite

$$
J_{p^{t+1}}(X) \stackrel{\left.J(f)\right|_{p_{p^{t+1}}(X)} ^{\longrightarrow}}{\longrightarrow} \Omega Y \stackrel{p^{r+t}}{\longrightarrow} \Omega Y
$$

is homotopic to the composite

$$
J_{p^{t+1}}(X) \stackrel{\text { pinch }}{\longrightarrow} X^{\left(p^{t+1}\right)} \stackrel{p^{r-1}\left(\sum_{\tau \in \Sigma_{p^{t+1}-1}} 1 \wedge \tau\right)}{\longrightarrow} X^{\left(p^{t+1}\right)} \stackrel{\widetilde{W}_{p^{t+1}}}{\longrightarrow} J(X) \stackrel{J(f)}{\longrightarrow} \Omega Y,
$$

where $p^{r+t}: \Omega Y \longrightarrow \Omega Y$ is the $p^{r+t}$-th power map, $\widetilde{W}_{n}$ is the $n$-fold Samelson product and

$1 \wedge \tau\left(x_{1} \wedge \cdots \wedge x_{p^{t+1}}\right)=x_{1} \wedge x_{\tau(2)} \wedge \cdots \wedge x_{\tau\left(p^{t+1}\right)}: X^{\left(p^{t+1}\right)} \longrightarrow X^{\left(p^{t+1}\right)}$

is the map which permutes positions. 
(3) Let $g=J(f) \circ \widetilde{W}_{p^{t+1}} \circ\left(\sum_{\tau \in \Sigma_{p^{t+1}-1}} 1 \wedge \tau\right) \circ p^{r-1}: X^{\left(p^{t+1}\right)} \longrightarrow \Omega Y$. This is an equivariant map with respect to the symmetric group action, that is,

$$
g \circ \sigma \simeq g
$$

for any $\sigma \in \Sigma_{p^{t+1}}$.

It is important to emphasize that parts (2) and (3) of Theorem 1.3 express the first nontrivial obstruction to the exactness of the Barratt Conjecture in terms of a computable equivariant map. Some special properties of the trace map $\Phi=\sum_{\tau \in \Sigma_{n}} \tau$ allows us to give a more detailed description of the first obstruction in the case when $X$ is a two-cell complex (see Proposition 4.4 and Theorem 4.6).

When originally formulated, the Barratt Conjecture did not have any example which supported it. The first known example that satisfies the statement of the Barratt Conjecture was the odd primary Moore space. Neisendorfer [7], following Cohen, Moore, and Neisendorfer's work [3] on the decomposition of the loop space on the mod $p^{r}$ Moore space, showed that $\Omega^{2} P^{n}\left(p^{r}\right)$ has multiplicative exponent $p^{r+1}$. When $p$ is an even prime, Theriault [10] showed that when $r \geq 6$ the Moore space $P^{n}\left(2^{r}\right)$ has exponent $2^{r+1}$. The existent of bounded exponent for the mod 2 Moore space remains a mystery. Our next goal is to find a property of $P^{n}(2)$ that will shed some light on the exponent problem.

Proposition 1.4 Let $X=P^{n}(2)$ be the $n$-dimensional mod 2 Moore space with $n \geq 3$. Then the composite

$$
X^{(3)} \stackrel{[2]}{\longrightarrow} X^{(3)} \stackrel{\sum_{\sigma \in \Sigma_{3}} \sigma}{\longrightarrow} X^{(3)}
$$

is null homotopic and therefore by Theorem 1.3 (2), the power map

$$
\left.8\right|_{J_{4}\left(P^{n}(2)\right)}: J_{4}\left(P^{n}(2)\right) \longrightarrow J\left(P^{n}(2)\right) \simeq \Omega\left(P^{n+1}(2)\right)
$$

is null homotopic.

The paper is organized as follows. In Section 2, we translate the algebraic model of [6] into geometry, establishing relations between combinatorial groups and the groups of natural transformations of a tensor algebra with the groups of homotopy classes of maps from the James construction to a loop space. Applications of this algebraic-geometric model to homotopy theory are given in Sections 3 and 4. In Section 3 we consider the Hopf invariant of the Whitehead product and prove Theorems 1.1 and 1.2. The exponent problem, with the emphasis on a rate of exponent growth for the Barratt Conjecture is 
treated in Section 4. In particular, in this section we describe some properties of the 8 -th power map on the mod 2 Moore space $P^{n}(2)$ proving Proposition 1.4.

Acknowledgements The authors would like to thank Professors John Berrick, Fred Cohen, Paul Selick and Stephen Theriault for their helpful suggestions and kind encouragement. The first author would also like to thank Professor John Berrick and the second author for making it possible for her to visit the National University of Singapore for a term and providing her with such a friendly working atmosphere.

\section{Geometrical realisations}

If $X$ is a connected $C W$ complex, the Bott-Samelson theorem says that $H_{*}(\Omega \Sigma X)$ is isomorphic as an algebra to $T\left(\widetilde{H}_{*}(X)\right)$, where $T$ denotes the tensor algebra. We can make $T\left(\widetilde{H}_{*}(X)\right)$ into a coalgebra by requiring that the elements of $\widetilde{H}_{*}(X)$ are primitive and then extend to all of $T\left(\widetilde{H}_{*}(X)\right)$ via the multiplication. If $X$ is itself a suspension, then $T\left(\widetilde{H}_{*}(X)\right)$ with this Hopf algebra structure is isomorphic as a Hopf algebra to $H_{*}(\Omega \Sigma X)$.

The James construction Let $X$ be a topological space with a non-degenerate basepoint $*$ and a compactly generated topology. Then the James construction $J(X)$ on $X$ is the free topological monoid generated by $X$ subject to the single relation that the basepoint $*$ is the unit. Combinatorially, the James construction $J(X)$ is obtained from the disjoint union $\bigsqcup_{k=1}^{\infty} X^{k}$ by identifying $\left(x_{1}, \ldots, x_{i}, \ldots, x_{k}\right)$ with $\left(x_{1}, \ldots, \hat{x}_{i}, \ldots, x_{k}\right)$ if $x_{i}=*$. Non-unit points of $J(X)$ can thus be thought of as words $\left(x_{1}, \ldots, x_{k}\right)$ of length $k$, with no $x_{i}$ being the unit. Let $q_{n}: X^{n} \longrightarrow J_{n}(X)$ be the quotient map. The James filtration $\left\{J_{n}(X)\right\}_{n \geq 0}$ with $J_{0}(X)=*$ and $J_{1}(X)=X$ is induced by the word-length filtration. It follows readily that $J_{n}(X) / J_{n-1}(X)$ is homeomorphic to $X^{(n)}$. The fundamental properties of $J(X)$ are as follows:

(1) if $X$ is path connected, then $J(X)$ is (weak) homotopy equivalent to $\Omega \Sigma X$;

(2) the quotient $\Sigma q_{n}: \Sigma X^{n} \longrightarrow \Sigma J_{n}(X)$ has a functorial cross-section;

(3) the inclusion $\Sigma J_{n-1}(X) \longrightarrow \Sigma J_{n}(X)$ has a functorial retraction;

(4) there is a functorial decomposition

$$
\Sigma J_{n}(X) \simeq \bigvee_{j=1}^{n} \Sigma X^{(j)}
$$

for $0 \leq n \leq \infty$. 
Recall that a space $X$ has a weak $L S$-category less than $k$ if the reduced diagonal $\bar{\Delta}_{k}: X \longrightarrow X^{(k)}$ is null homotopic. The spaces that we will consider in the following sections will be path connected and will have a weak $L S$-category less than 2, that is, the reduced diagonal $\bar{\Delta}: X \longrightarrow X \wedge X$ is null homotopic (for example, if $X$ is a co- $H$ space).

Geometrical Cohen groups Let $X$ be a pointed path connected space, $X^{n}$ the $n-$ fold self Cartesian product of $X$ and $X^{(k)}$ the $k$-fold self smash product of $X$. Let $J(X)$ be the James construction of $X$. The following groups were introduced by Cohen in [2] and $\mathrm{Wu}[12]$.

Definition 2.1 Let $K_{n}(X)$ denote the subgroup of $\left[X^{n}, J(X)\right]$ generated by the homotopy classes $x_{i}$ for $1 \leq i \leq n$, where $x_{i}$ is represented by the composite

$$
X^{n} \stackrel{p_{i}}{\longrightarrow} X \stackrel{E}{\longrightarrow} J(X)
$$

where $p_{i}: X^{n} \longrightarrow X$ is the $i$-th coordinate projection given by

$$
p_{i}\left(x_{1}, x_{2}, \cdots, x_{n}\right)=x_{i},
$$

and $E: X \longrightarrow J(X)$ is the canonical inclusion.

Proposition 2.2 (Cohen [2]) Let $X$ be a path connected space with weak $L S$ category less than 2 . Then, in the group $\left[X^{n}, J X\right]$, the following identities hold:

(1) $\left[\left[x_{i_{1}}, x_{i_{2}}, \cdots, x_{i_{k}}\right]=1\right.$ if $i_{s}=i_{t}$ for some $1 \leq s<t \leq k$, where $\left[\left[a_{1}, a_{2}, \cdots, a_{l}\right]=\left[\cdots\left[a_{1}, a_{2}\right], \cdots,\right], a_{l}\right]$ with $[x, y]=x^{-1} y^{-1} x y$;

(2) $\left[\left[x_{i_{1}}^{n_{1}}, x_{i_{2}}^{n_{2}}, \cdots, x_{i_{k}}^{n_{l}}\right]=\left[\left[x_{i_{1}}, x_{i_{2}}, \cdots, x_{i_{k}}\right]^{n_{1} n_{2} \cdots n_{l}}\right.\right.$.

Recall that the Cohen group $K_{n}$ is defined combinatorially as follows. The Cohen group $K_{n}\left(x_{1}, x_{2}, \ldots, x_{n}\right)$ is the quotient group of the free group $F_{n}$ of rank $n$ generated by $x_{1}, x_{2}, \ldots, x_{n}$ modulo the relations

(1) $\left[\left[x_{i_{1}}, x_{i_{2}}, x_{i_{3}}, \cdots, x_{i_{l}}\right]=1\right.$ if $i_{s}=i_{t}$ for some $1 \leq s, t \leq l$;

(2) $\left[\left[x_{i_{1}}^{n_{1}}, x_{i_{2}}^{n_{2}}, x_{i_{3}}^{n_{3}}, \cdots, x_{i_{l}}^{n_{l}}\right]=\left[\left[x_{i_{1}}, x_{i_{2}}, x_{i_{3}}, \cdots, x_{i_{l}}\right]^{n_{1} n_{2} \cdots n_{l}}\right.\right.$.

Proposition 2.3 Let $X$ be a path connected space with weak $L S$-category less than 2 . Then there is a homomorphism

$$
e_{X}: K_{n} \longrightarrow K_{n}(X) \subseteq\left[X^{n}, J(X)\right]
$$

given on any generator $x_{i}$ of $K_{n}$ by $e_{X}\left(x_{i}\right)=x_{i}$. 
Proof The existence of the homomorphism $e_{X}$ follows readily from the definition of $K_{n}$ and Proposition 2.2.

This homomorphism can be generalized in the following way.

Corollary 2.4 Let $X$ be a path connected space with weak $L S$-category less than 1, $M$ a path connected topological monoid and $f: X \longrightarrow M$ a pointed map. Then there is homomorphism

given by

$$
e_{f}: K_{n} \longrightarrow\left[X^{n}, M\right]
$$

$$
e_{f}\left(x_{i}\right)=J(f)_{*}\left(x_{i}\right)
$$

where $J(f): J\left(X^{(k)}\right) \longrightarrow M$ is a homomorphism of topological monoids such that $\left.J(f)\right|_{X}=f$ and $J(f)_{*}:\left[X^{n}, J(X)\right] \longrightarrow\left[X^{n}, M\right]$ is induced by the map $J(f)$.

Proposition 2.5 (Cohen [2]) Let $X$ be a path connected space with weak $L S$ category less than 1, $M$ a path-connected topological monoid and $f: X \longrightarrow M$ a pointed map. Suppose that the $q$-th power $[f]^{q}=1$ in the group $[X, M]$. Then the homomorphism $\theta_{f}: K_{n} \longrightarrow\left[X^{n}, M\right]$ factors through the quotient group $K_{n}^{\mathbb{Z} / q}$.

\section{Generalization to $K_{n}(k)(X)$}

Definition 2.6 Let $k \leq n$. Define $K_{n}(k)(X)$ to be the subgroup of $\left[X^{n}, J\left(X^{(k)}\right)\right]$ generated by the homotopy classes $\left\{x_{i_{1}}\left|x_{i_{2}}\right| \cdots \mid x_{i_{k}}\right\}$ for $1 \leq i_{j} \leq n$ and $1 \leq j \leq k$, where $\left\{x_{i_{1}}\left|x_{i_{2}}\right| \cdots \mid x_{i_{k}}\right\}$ is represented by the composite

$$
X^{n} \stackrel{p_{i_{1} \cdots i_{k}}}{\longrightarrow} X^{(k)} \stackrel{E}{\longrightarrow} J\left(X^{(k)}\right),
$$

where $E: X^{(k)} \longrightarrow J\left(X^{(k)}\right)$ is the canonical inclusion and $p_{i_{1} \cdots i_{k}}: X^{n} \longrightarrow X^{(k)}$ is given by

$$
p_{i_{1} \cdots i_{k}}\left(x_{1}, x_{2}, \cdots, x_{n}\right)=x_{i_{1}} \wedge \cdots \wedge x_{i_{k}}
$$

Notice that in the case $k=1$, the group $K_{n}(1)(X)$ is the Cohen group $K_{n}(X)$.

Proposition 2.7 (Lemma 2.2, [12]) Let $X$ be a path connected space with weak $L S$-category less than 2. Then, in the group $\left[X^{n}, J\left(X^{(k)}\right)\right]$, the following identities hold:

(1) $\left\{x_{i_{1}}\left|x_{i_{2}}\right| \cdots \mid x_{i_{k}}\right\}=1 \quad$ if $i_{s}=i_{t}$ for some $1 \leq s<t \leq k$; 
(2)

$$
\begin{gathered}
\text { (2) }\left[\left[\left\{x_{i_{1}}\left|x_{i_{2}}\right| \cdots \mid x_{i_{k}}\right\},\left\{x_{i_{k+1}}\left|x_{i_{k+2}}\right| \cdots \mid x_{i_{2 k}}\right\}, \cdots\right.\right. \\
\left.\cdots,\left\{x_{i_{(l-1) k+1}}\left|x_{i_{(l-1) k+2}}\right| \cdots \mid x_{i_{l k}}\right\}\right]=1 \\
\text { if } i_{s}=i_{t} \text { for some } 1 \leq s<t \leq k l, \text { where }\left[\left[a_{1}, a_{2}, \cdots, a_{l}\right]=\left[\cdots\left[a_{1}, a_{2}\right], \cdots,\right], a_{l}\right] \\
\text { with }[x, y]=x^{-1} y^{-1} x y ; \\
\text { (3) }\left[\left[\left\{x_{i_{1}}\left|x_{i_{2}}\right| \cdots \mid x_{i_{k}}\right\}^{n_{1}},\left\{x_{i_{k+1}}\left|x_{i_{k+2}}\right| \cdots \mid x_{i_{2 k}}\right\}^{n_{2}, \cdots}, \cdots\right.\right. \\
\left.\cdots,\left\{x_{i_{(l-1) k+1}}\left|x_{i_{(l-1) k+2}}\right| \cdots \mid x_{i_{l k}}\right\}^{n_{l}}\right]= \\
{\left[\left[\left\{x_{i_{1}}\left|x_{i_{2}}\right| \cdots \mid x_{i_{k}}\right\},\left\{x_{i_{k+1}}\left|x_{i_{k+2}}\right| \cdots \mid x_{i_{2 k}}\right\}, \cdots\right.\right.} \\
\left.\cdots,\left\{x_{i_{(l-1) k+1}}\left|x_{i_{(l-1) k+2}}\right| \cdots \mid x_{i_{l k}}\right\}\right]_{.}^{n_{1} n_{2} \cdots n_{l}}
\end{gathered}
$$

Remark Relation (3) follows from relation (2) (see for example [2]).

Recall from [6] that the group $K_{n}(k)$ is defined combinatorially as follows:

(1) generators are the words $\left\{x_{i_{1}}\left|x_{i_{2}}\right| \cdots \mid x_{i_{k}}\right\}$ with $1 \leq i_{j} \leq n$ for $1 \leq j \leq k$;

(2) relations are given by identities (1) - (3) in Proposition 2.7.

Let $q$ be an integer. The group $K_{n}^{\mathbb{Z} / q}(k)$ is the quotient group of $K_{n}(k)$ modulo the following additional relations:

(3) $\left\{x_{i_{1}}\left|x_{i_{2}}\right| \cdots \mid x_{i_{k}}\right\}^{q}=1$ for each generator $\left\{x_{i_{1}}\left|x_{i_{2}}\right| \cdots \mid x_{i_{k}}\right\}$.

Proposition 2.8 Let $X$ be a path connected space with weak $L S$-category less than 2 . Then there is a homomorphism

$$
e_{X}: K_{n}(k) \longrightarrow\left[X^{n}, J\left(X^{(k)}\right)\right]
$$

given on any generator $\left\{x_{i_{1}}\left|x_{i_{2}}\right| \cdots \mid x_{i_{k}}\right\}$ of $K_{n}(k)$ by

$$
e_{X}\left(\left\{x_{i_{1}}\left|x_{i_{2}}\right| \cdots \mid x_{i_{k}}\right\}\right)=\left\{x_{i_{1}}\left|x_{i_{2}}\right| \cdots \mid x_{i_{k}}\right\} \text {. }
$$

Proof The existence of the homomorphism $e_{X}$ follows immediately from the definition of $K_{n}(k)$ and Proposition 2.7.

Corollary 2.9 Let $X$ be a path connected space with weak $L S$-category less than 2, $M$ be a path connected topological monoid and $f: X^{(k)} \longrightarrow M$ a pointed map. By the definition of $K_{n}(k)$, the homomorphism

$$
\begin{gathered}
e_{f}: K_{n}(k) \longrightarrow\left[X^{n}, M\right], \\
\text { given by } \quad e_{f}\left(\left\{x_{i_{1}}\left|x_{i_{2}}\right| \cdots \mid x_{i_{k}}\right\}\right)=J(f)_{*}\left(\left\{x_{i_{1}}\left|x_{i_{2}}\right| \cdots \mid x_{i_{k}}\right\}\right),
\end{gathered}
$$

is well-defined, where $J(f): J\left(X^{(k)}\right) \longrightarrow M$ is a homomorphism of topological monoids such that $\left.J(f)\right|_{X^{(k)}}=f$ and $J(f)_{*}:\left[X^{n}, J\left(X^{(k)}\right)\right] \longrightarrow\left[X^{n}, M\right]$ is induced by the map $J(f)$. 
Lemma 2.10 Let $X$ be a path connected space with weak $L S$-category less than 2, $M$ a path connected topological monoid and $f: X^{(k)} \longrightarrow M$ a pointed map. Suppose that the $q$-th power $[f]^{q}=1$ in the group $\left[X^{(k)}, M\right]$. Then the homomorphism $e_{f}: K_{n}(k) \longrightarrow\left[X^{n}, M\right]$ factors through the quotient group $K_{n}^{\mathbb{Z} / q}(k)$.

Proof The image under $e_{f}$ of the element $\left\{x_{i_{1}}\left|x_{i_{2}}\right| \cdots \mid x_{i_{k}}\right\}^{q}$ is represented by the composite

$$
X^{n} \stackrel{p_{i_{1} \cdots i_{k}}}{\longrightarrow} X^{(k)} \stackrel{E}{\longrightarrow} J\left(X^{(k)}\right) \stackrel{J(f)}{\longrightarrow} J(M) \stackrel{q}{\longrightarrow} J(M) \longrightarrow M .
$$

As $[f]^{q}=1$, the composite is null homotopic. Thus the assertion follows.

Generalization to $\mathcal{H}_{n}(X)$ By the suspension splitting theorem (2-1), the inclusion

$$
J_{n-1}(X) \longrightarrow J_{n}(X)
$$

induces a tower of group epimorphisms

$$
[J(X), \Omega Y] \longrightarrow \cdots \longrightarrow\left[J_{n}(X), \Omega Y\right] \longrightarrow \cdots \longrightarrow[X, \Omega Y]
$$

and there is a group isomorphism between $[J(X), \Omega Y]$ and the inverse limit

$$
[J(X), \Omega Y] \cong \lim _{n}\left[J_{n}(X), \Omega Y\right]
$$

Let $q_{n}: X^{n} \longrightarrow J_{n}(X)$ be the quotient map. Then by the suspension splitting theorem $(2-1)$, there is a group monomorphism

$$
q_{n}^{*}:\left[J_{n}(X), J(X)\right] \longrightarrow\left[X^{n}, J(X)\right]
$$

for each $n$.

Definition 2.11 Let $\mathcal{H}_{n}(X)$ be the subgroup of $\left[J_{n}(X), J(X)\right]$ defined by

$$
\left[J_{n}(X), J(X)\right] \cap K_{n}(X) .
$$

There is an equivalent definition for the groups $\mathcal{H}_{n}(X)$ which is more suitable for generalization. Recall that $J_{n}(X)$ is the coequalizer of the inclusions $i_{j}: X^{n-1} \longrightarrow X^{n}$ for $1 \leq j \leq n$. These inclusions induce projections

$$
i_{j}^{*}:\left[X^{n}, Y\right] \longrightarrow\left[X^{n-1}, Y\right]
$$

for $1 \leq j \leq n$ and any space $Y$. Thus there are projections $d_{j}: K_{n}(X) \longrightarrow K_{n-1}(X)$ given by

$$
d_{j}\left(x_{i}\right)=\left\{\begin{array}{ll}
x_{i} & \text { for } i<j \\
1 & \text { for } i=j \\
x_{i-1} & \text { for } i>j
\end{array} \quad \text { for } 1 \leq j \leq n\right.
$$


Lemma 2.12 The diagram of group homomorphisms

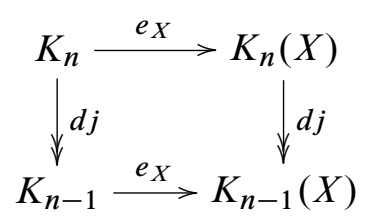

commutes for every $1 \leq j \leq n$.

Proof The proof follows immediately from the definitions.

Define the group $\mathcal{H}_{n}(X)$ to be the equalizer of homomorphisms $d_{j}$ for $1 \leq j \leq n$. From the above discussion, it follows readily that the two definitions of $\mathcal{H}_{n}(X)$ are equivalent. Now from the second definition of $\mathcal{H}_{n}(X)$, as $\left.d_{i}\right|_{\mathcal{H}_{n}(X)}=\left.d_{j}\right|_{\mathcal{H}_{n}(X)}$ for $1 \leq i, j \leq n$, there are homomorphisms $p_{n}: \mathcal{H}_{n}(X) \longrightarrow \mathcal{H}_{n-1}(X)$ such that the diagram

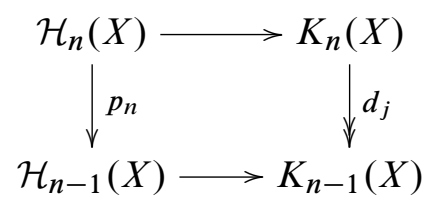

commutes for $1 \leq j \leq n$. Selick and Wu [8] proved that there is a progroup

$$
\mathcal{H}_{\infty}(X) \longrightarrow \cdots \longrightarrow \mathcal{H}_{n}(X) \longrightarrow \cdots \longrightarrow \mathcal{H}_{0}(X)
$$

where $\mathcal{H}_{\infty}(X)$ is given by the inverse limit

$$
\mathcal{H}_{\infty}(X)=\lim _{n} \mathcal{H}_{n}(X)
$$

In [6] we recalled the combinatorial group $\mathcal{H}_{n}$ as the equalizer of the projections $p_{j}: K_{n} \longrightarrow K_{n-1}$ for $1 \leq j \leq n$. The definition of $\mathcal{H}_{n}$ in this setting goes back to Cohen [2].

Proposition 2.13 Let $X$ be a path connected space with weak $L S$-category less than 2. Then there is a homomorphism

$$
e_{X}: \mathcal{H}_{n} \longrightarrow \mathcal{H}_{n}(X) \subseteq\left[J_{n}(X), J(X)\right]
$$


such that the diagram

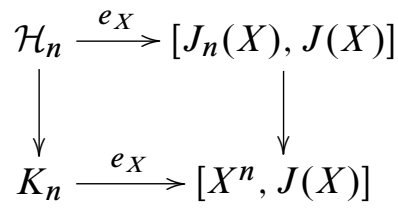

commutes.

Proof Notice that the group $\left[J_{n}(X), J(X)\right]$ can be thought of as the equalizer of the projections $i_{j}^{*}:\left[X^{n}, J(X)\right] \longrightarrow\left[X^{n-1}, J(X)\right]$. The existence of the homomorphism $e_{X}: \mathcal{H}_{n} \longrightarrow\left[J_{n}(X), J(X)\right]$ follows readily from the existence of $e_{X}: K_{n} \longrightarrow$ $K_{n}(X) \subseteq\left[X^{n}, J(X)\right]$, the fact that the groups $\mathcal{H}_{n}$ and $\mathcal{H}_{n}(X)$ are the equalizers of appropriate projections on $K_{n}$ and $K_{n}(X)$, respectively , and that diagram (2-2) commutes.

Corollary 2.14 Let $X$ be a path connected space with weak $L S$-category less than 2, $M$ be a path connected topological monoid and $f: X \longrightarrow M$ a pointed map. Then there is a homomorphism

$$
e_{f}: \mathcal{H}_{n} \longrightarrow\left[J_{n}(X), M\right]
$$

given by $e_{f}=J(f)_{*} \circ e_{X}$.

Corollary 2.15 Let $X$ be a path connected space with weak $L S$-category less than 2, $M$ a path connected topological monoid and $f: X \longrightarrow M$ a pointed map. Suppose that the $q$-th power $[f]^{q}=1$ in the group $[X, M]$. Then the homomorphism $e_{f}: \mathcal{H}_{n} \longrightarrow$ $\left[J_{n}(X), M\right]$ factors through the quotient group $\mathcal{H}_{n}^{\mathbb{Z} / q}$.

Generalization to $\mathcal{H}_{n}^{(l)}(X)$ Further on, we want to find a geometrical analogue of the combinatorial group $\mathcal{H}_{n}^{(l)}$ defined in [6, Definition 2.7].

Proposition 2.16 The group $\left[J_{n}\left(X^{(l)}\right), J(X)\right]$ is the equalizer of the restriction of the projections $D_{j}: K_{l n}(X) \longrightarrow K_{l(n-1)}(X)$, that is,

$$
\begin{gathered}
K_{l n}(X) \cap\left(\bigcap_{s=0}^{n-1}\left(\bigcap_{j=1}^{l} \operatorname{Ker} i_{s l+1}^{*}\right)\right) \\
D_{0} \downarrow{ } \cdot . D_{n-1}^{D_{n-1}} \\
K_{l(n-1)}(X) \cap\left(\bigcap_{s=0}^{n-2}\left(\bigcap_{j=1}^{l} \operatorname{Ker} i_{s l+1}^{*}\right)\right)
\end{gathered}
$$


where the projection $D_{j}$ is given by

$$
D_{j}\left(x_{i}\right)= \begin{cases}x_{i} & \text { for } i<l j \\ 1 & \text { for } l j+1 \leq i \leq(l+1) j \\ x_{i-1} & \text { for }(l+1) j<i\end{cases}
$$

for $0 \leq j \leq n-1$.

Proof Notice that the composite

$$
X^{l n} \longrightarrow \underbrace{X^{(l)} \times \cdots \times X^{(l)}}_{n} \longrightarrow J_{n}\left(X^{(l)}\right)
$$

induces a group monomorphism

$$
\left[J_{n}\left(X^{(l)}\right), J(X)\right] \longrightarrow[\underbrace{X^{(l)} \times \cdots \times X^{(l)}}_{n}, J(X)] \longrightarrow\left[X^{l n}, J(X)\right] .
$$

Let $d_{j}$ denote the following projection

$$
\underbrace{X^{(l)} \times \cdots \times X^{(l)}}_{n} \longrightarrow \underbrace{X^{(l)} \times \cdots \times \widehat{X^{(l)}} \cdots \times X^{(l)}}_{n-1}
$$

for $1 \leq j \leq n$.

Let $i_{j}: X^{l} \longrightarrow X^{l+1}$ denote the usual coordinate inclusion

$$
i_{j}\left(x_{1}, \ldots, x_{l}\right)=\left(x_{1}, \ldots, x_{j-1}, *, x_{j}, \ldots x_{l}\right) .
$$

Notice that if

$$
\alpha \in\left[X^{l n}, J(X)\right] \cap[\underbrace{X^{(l)} \times \cdots \times X^{(l)}}_{n}, J(X)],
$$

then

$$
\alpha \in \bigcap_{s=0}^{n-1}\left(\bigcap_{j=1}^{l} \operatorname{Ker} i_{s l+j}^{*}\right) .
$$

Now it is clear that $\left[J_{n}\left(X^{(l)}\right), J(X)\right]$ is

$$
\operatorname{eq}\left(D_{0}, \ldots, D_{n-1}\right) \bigcap\left(\bigcap_{s=0}^{n-1}\left(\bigcap_{j=1}^{l} \operatorname{Ker} i_{s l+j}^{*}\right)\right)
$$

where eq $\left(f_{1}, \ldots, f_{n}\right)$ stands for the equalizer of maps $f_{1}, \ldots, f_{n}$.

Algebraic $8 \mathcal{G}$ Geometric Topology, Volume 6 (2006) 
Definition 2.17 Define the group $\mathcal{H}_{n}^{(l)}(X)$ to be the subgroup of $\left[J_{n}\left(X^{(l)}\right), J(X)\right]$ given by the equalizer of the projections

$$
\begin{gathered}
K_{l n}(X) \cap\left(\bigcap_{s=0}^{n-1}\left(\bigcap_{j=1}^{l} \operatorname{Ker} i_{s l+1}^{*}\right)\right) \\
D_{0} \mid \cdot \cdot \downarrow^{D_{n-1}} \\
K_{l(n-1)}(X) \cap\left(\bigcap_{s=0}^{n-2}\left(\bigcap_{j=1}^{l} \operatorname{Ker} i_{s l+1}^{*}\right)\right) .
\end{gathered}
$$

From the definition of $\mathcal{H}_{n}^{(l)}(X)$, as $\left.D_{i}\right|_{\mathcal{H}_{n}^{(l)}(X)}=\left.D_{j}\right|_{\mathcal{H}_{n}^{(l)}(X)}$ for every $1 \leq i, j \leq n$, there is a homomorphism $p_{n}: \mathcal{H}_{n}^{(l)}(X) \longrightarrow \mathcal{H}_{n-1}^{(l)}(X)$ such that the diagram

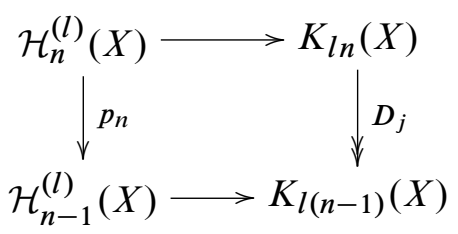

commutes for $0 \leq j \leq n-1$.

Lemma 2.18 There is a progroup

$$
\mathcal{H}^{(l)}(X) \longrightarrow \cdots \longrightarrow \mathcal{H}_{n}^{(l)}(X) \longrightarrow \cdots \longrightarrow \mathcal{H}_{0}^{(l)}(X)
$$

where $\mathcal{H}^{(l)}(X)$ is given by the inverse limit

$$
\mathcal{H}^{(l)}(X)=\lim _{p_{n}} \mathcal{H}_{n}^{(l)}(X) .
$$

Proof The map $p_{n}: \mathcal{H}_{n}^{(l)}(X) \longrightarrow \mathcal{H}_{n-1}^{(l)}(X)$ is obtained as the map of equalizers induced by the epimorphisms $D_{j}$. Thus $p_{n}$ is an epimorphism for every $n>0$.

Proposition 2.19 For every $n>0$, there is a homomorphism

$$
e_{X}: \mathcal{H}_{n}^{(l)} \longrightarrow \mathcal{H}_{n}^{(l)}(X) \subseteq\left[J_{n}\left(X^{(l)}\right), J(X)\right]
$$

such that the diagram

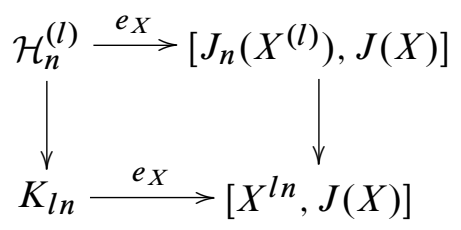

commutes. 
Proof The statement follows immediately from the definition of the groups $\mathcal{H}_{n}^{(l)}$ and $\mathcal{H}_{n}^{(l)}(X)$ and the existence of the homomorphism $e_{X}: K_{l n} \rightarrow\left[X^{l n}, J(X)\right]$.

Corollary 2.20 Let $X$ be a path connected space with weak $L S$-category less than 1, $M$ be a path connected topological monoid and $f: X \longrightarrow M$ a pointed map. Then there is a homomorphism

$$
e_{f}: \mathcal{H}_{n}^{(l)} \longrightarrow\left[J_{n}\left(X^{(l)}\right), M\right]
$$

given by $e_{f}=J(f)_{*} \circ e_{X}$.

Corollary 2.21 Let $X$ be a path connected space with weak $L S$-category less than 2, $M$ a path connected topological monoid and $f: X \longrightarrow M$ a pointed map. Suppose that the $q$-th power $[f]^{q}=1$ in the group $[X, M]$. Then the homomorphism $e_{f}: \mathcal{H}_{n}^{(l)} \longrightarrow$ $\left[J_{n}\left(X^{(l)}\right), M\right]$ factors through the quotient group $\mathbb{Z} / q \mathcal{H}_{n}^{(l)}$.

Generalization to $\mathcal{H}_{n}^{(l)(k)}(X)$

Definition 2.22 Define the group $\mathcal{H}_{n}^{(l)(k)}(X)$ to be the subgroup of the group $\left[J_{n}\left(X^{(l)}\right), J\left(X^{(k)}\right)\right]$ given by the equalizer of the projections

$$
\begin{gathered}
K_{l n}(k)(X) \cap\left(\bigcap_{s=0}^{n-1}\left(\bigcap_{j=1}^{l} \operatorname{Ker} i_{s l+1}^{*}\right)\right) \\
D_{0} \downarrow \cdot \cdot \downarrow D_{n-1} \\
K_{l(n-1)}(k)(X) \cap\left(\bigcap_{s=0}^{n-2}\left(\bigcap_{j=1}^{l} \operatorname{Ker} i_{s l+1}^{*}\right)\right) .
\end{gathered}
$$

where the homomorphisms $D_{i}$ are induced by those from Definition 2.17.

From the definition of $\mathcal{H}_{n}^{(l)(k)}(X)$, as $\left.D_{i}\right|_{\mathcal{H}_{n}^{(l)(k)}(X)}=\left.D_{j}\right|_{\mathcal{H}_{n}^{(l)(k)}(X)}$ for every $1 \leq$ $i, j \leq n$, there is a homomorphism $p_{n}: \mathcal{H}_{n}^{(l)(k)}(X) \longrightarrow \mathcal{H}_{n-1}^{(l)(k)}(X)$ such that the following diagram

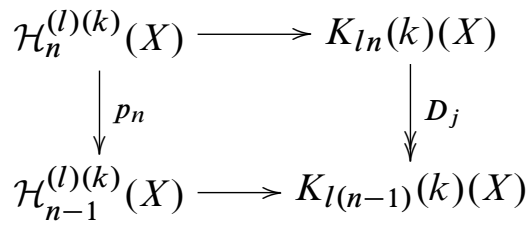

commutes for $0 \leq j \leq n-1$. 
Lemma 2.23 There is a progroup

$$
\mathcal{H}^{(l)(k)}(X) \longrightarrow \cdots \longrightarrow \mathcal{H}_{n}^{(l)(k)}(X) \longrightarrow \cdots \longrightarrow \mathcal{H}_{0}^{(l)(k)}(X)
$$

where $\mathcal{H}^{(l)(k)}(X)$ is given by the inverse limit

$$
\mathcal{H}^{(l)(k)}(X)=\lim _{p_{n}} \mathcal{H}_{n}^{(l)(k)}(X) .
$$

Proof The map $p_{n}: \mathcal{H}_{n}^{(l)(k)}(X) \longrightarrow \mathcal{H}_{n-1}^{(l)(k)}(X)$ is obtained as the map of equalizers induced by the epimorphisms $D_{j}$. Thus $p_{n}$ is an epimorphism for every $n>0$.

The combinatorial group $\mathcal{H}_{n}^{(l)(k)}$ is defined in the predecessor paper [6, Definition 2.9].

Proposition 2.24 There is a homomorphism

$$
e_{X}: \mathcal{H}_{n}^{(l)(k)} \longrightarrow \mathcal{H}_{n}^{(l)(k)}(X) \subseteq\left[J_{n}\left(X^{(l)}\right), J\left(X^{(k)}\right)\right]
$$

such that the diagram

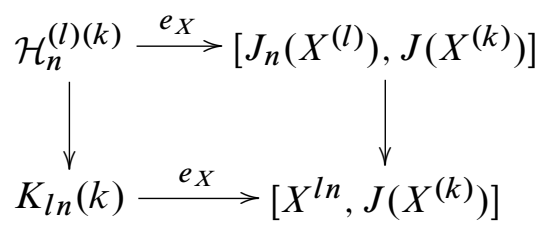

commutes.

Proof The statement follows immediately from the definition of the group $\mathcal{H}^{(l)(k)}(X)$ and the existence of the homomorphism $e_{X}: K_{l n}(k) \rightarrow\left[X^{l n}, J\left(X^{(k)}\right)\right]$.

Corollary 2.25 Let $X$ be a path connected space with weak $L S$-category less than 2, $M$ be a path connected topological monoid and $f: X^{(k)} \longrightarrow M$ a pointed map. Then there is a homomorphism

$$
e_{f}: \mathcal{H}_{n}^{(l)(k)} \longrightarrow\left[J_{n}\left(X^{(l)}\right), M\right]
$$

given by $e_{f}=J(f)_{*} \circ e_{X}$.

Corollary 2.26 Let $X$ be a path connected space with weak $L S$-category less than 2, $M$ a path connected topological monoid and $f: X^{(k)} \longrightarrow M$ a pointed map. Suppose that the $q$-th power $[f]^{q}=1$ in the group $\left[X^{(k)}, M\right]$. Then the homomorphism $e_{f}: \mathcal{H}_{n}^{(l)(k)} \longrightarrow\left[J_{n}\left(X^{(l)}\right), M\right]$ factors through the quotient group $\mathbb{Z} / q \mathcal{H}_{n}^{(l)(k)}$. 


\section{Application: Whitehead products and James-Hopf maps}

James-Hopf maps Let $X$ be a pointed space. The James-Hopf map

$$
H_{k}: J(X) \longrightarrow J\left(X^{(k)}\right)
$$

is combinatorially defined by

$$
H_{k}\left(x_{1} x_{2} \ldots x_{n}\right)=\prod_{1 \leq i_{1}<i_{2}<\cdots<i_{k} \leq n}\left(x_{i_{1}} x_{i_{2}} \ldots x_{i_{k}}\right)
$$

with right lexicographical order in the product.

The James-Hopf map can be also defined more geometrically. Let $X$ have a homotopy type of a $C W$ complex. Then using the simple combinatorial structure of the James construction $J(X)$, there can be made a preferred choice (inductively on the James filtration $\left.J_{n}(X)\right)$ of the homotopy equivalence

$$
\theta: \Sigma(J(X)) \simeq \Sigma \Omega \Sigma X \longrightarrow \bigvee_{i=0}^{\infty} \Sigma X^{(i)} .
$$

Furthermore, consider the adjoint of $\theta$

$$
\bar{\theta}: \Omega \Sigma X \longrightarrow \Omega\left(\bigvee_{i=0}^{\infty} \Sigma X^{(i)}\right),
$$

and the pinch map

$$
q_{k}: \bigvee_{i=0}^{\infty} X^{(i)} \longrightarrow X^{(k)}
$$

which sends $X^{(i)}$ to the base point if $i \neq k$ and which is the identity when restricted to $X^{(k)}$. Now, the $k$-th James-Hopf map

$$
H_{k}: \Omega \Sigma X \longrightarrow \Omega \Sigma X^{(k)}
$$

is defined as the composite $\Omega \Sigma\left(q_{k}\right) \circ \bar{\theta}$.

In this paper we shall take advantage of the combinatorial definition of the James-Hopf map.

For each $n$, let $H_{k *}:\left[X^{n}, J(X)\right] \longrightarrow\left[X^{n}, J\left(X^{(k)}\right)\right]$ be the function induced by the map $H_{k}$. Notice that $H_{k *}$ is not a homomorphism of groups if $n \geq k>1$ and $X$ is a non-contractible suspension. We want to study the map $H_{k *}$ using the combinatorial methods described in Section 1, namely, the relation between groups $K_{n}$ and $K_{n}(k)$ 
and the natural maps from $X^{n}$ to $J(X), J\left(X^{(k)}\right)$, respectively. Therefore we proceed by defining the combinatorial analogue

$$
H_{k}: K_{n}=K_{n}(1) \longrightarrow K_{n}(k)
$$

of $H_{k *}$.

Definition 3.1 The function $H_{k}: K_{n}=K_{n}(1) \longrightarrow K_{n}(k)$ is defined by setting

$$
H_{k}\left(x_{i_{1}}^{n_{1}} x_{i_{2}}^{n_{2}} \cdots x_{i_{l}}^{n_{l}}\right)=\prod_{1 \leq j_{1}<\cdots<j_{k} \leq l}\left\{x_{i_{j_{1}}}\left|x_{i_{j_{2}}}\right| \cdots \mid x_{i_{j_{k}}}\right\}^{n_{j_{1}} n_{j_{2}} \cdots n_{j_{k}}}
$$

with right lexicographical order, for any word $x_{i_{1}}^{n_{1}} x_{i_{2}}^{n_{2}} \cdots x_{i_{l}}^{n_{l}} \in K_{n}$.

Proposition 3.2 [12, Lemma 2.3] The function $H_{k}: K_{n} \longrightarrow K_{n}(k)$ is well-defined. Furthermore, there is a commutative diagram

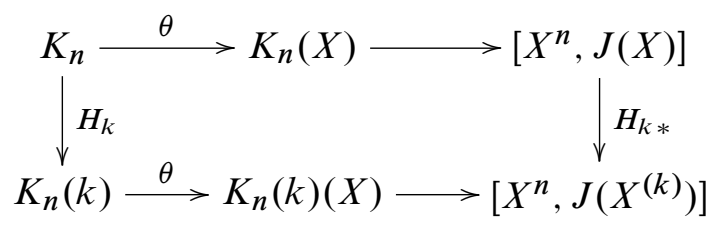

for any suspension $X$.

The algebraic analogue of the James-Hopf map $H_{k}: T(V) \longrightarrow T\left(V^{\otimes k}\right)$ is the functorial coalgebra map induced from

$$
H_{k *}: H_{*}(\Omega \Sigma X) \longrightarrow H_{*}\left(\Omega \Sigma\left(X^{(k)}\right)\right) .
$$

Lemma 3.3 The function $H_{k}: K_{n t} \longrightarrow K_{n t}(k)$ induces a function

$$
h_{k}: \mathcal{H}_{t}^{(n)} \longrightarrow \mathcal{H}_{t}^{(n),(k)}
$$

for any $1 \leq t \leq \infty$. Moreover there is a commutative diagram

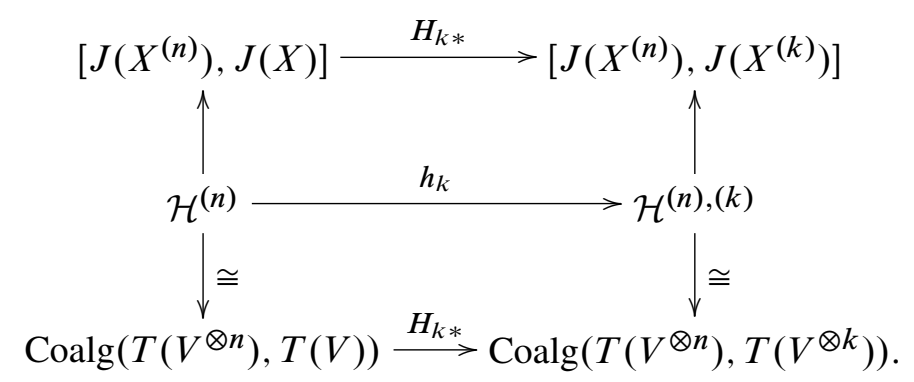

Algebraic 83 Geometric Topology, Volume 6 (2006) 
Proof The first statement follows from the direct computation and the fact that $\mathcal{H}_{t}^{(n)}$ and $\mathcal{H}_{t}^{(n),(k)}$ are given by certain equalizers [6, Definitions $2.7,2.9$ ].

The top square of the diagram commutes by Proposition 3.2. In paper [6], we established the progroup isomorphisms $e: \mathcal{H}^{(n)} \longrightarrow \operatorname{Coalg}\left(T\left(V^{\otimes n}\right), T(V)\right)$ and $e: \mathcal{H}^{(n),(k)} \longrightarrow$ Coalg $\left(T\left(V^{\otimes n}\right), T\left(V^{\otimes k}\right)\right)$. By applying the homology functor, it follows that

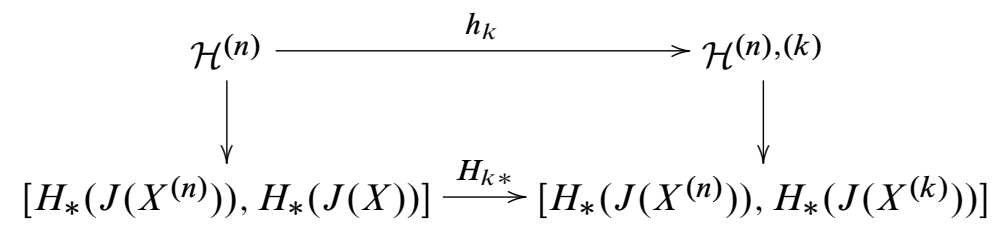

and hence the bottom square commutes by letting $X$ run through wedges of the 2sphere.

The main objective of this section is the study of the Hopf invariant of a Whitehead product. Let $W_{n}: \Sigma X^{(n)} \longrightarrow \Sigma X$ denote the $n$ fold Whitehead product on $X$. Our main result is as follows.

Theorem 3.4 Let $X$ be a pointed space with the null homotopic reduced diagonal $\bar{\Delta}: X \longrightarrow X \wedge X$. Then for $n>k$,

$$
J\left(X^{(n)}\right) \stackrel{\Omega W_{n}}{\longrightarrow} J(X) \stackrel{H_{k}}{\longrightarrow} J\left(X^{(k)}\right)
$$

is null homotopic if $k$ does not divide $n$.

Proof We start by calculating the algebraic analogue of the $k$-th Hopf invariant of the $n$-fold Whitehead product and then transfer the result to topology. The geometrical map $H_{k} \circ \Omega W_{n}$ induces the map

$$
H_{k} \circ T\left(\beta_{n}\right): T\left(V^{\otimes n}\right) \longrightarrow T\left(V^{\otimes k}\right) .
$$

Wu [12] proved that the map $H_{k} \circ \Omega \omega_{n}$ is a loop map. Therefore the induced map $H_{k} \circ T\left(\beta_{n}\right)$ is an algebra map which is determined by its values on $V^{\otimes n}$. If $n$ in not a multiple of $k$, then because of dimensional reasons $H_{k} \circ \beta_{n}=0$, that is, $\left.H_{k} \circ T\left(\beta_{n}\right)\right|_{V \otimes n}=0$. That shows that $H_{k} \circ T\left(\beta_{n}\right)=0$. Now applying diagram (3-1) proves the theorem.

Theorem 3.5 For $X$ as in Theorem 3.4, define the map $\Phi: \Sigma X^{(4)} \longrightarrow \Sigma X^{(4)}$ as

$$
\Phi=\tau_{34}-\tau_{12} \tau_{34}+\tau_{14} \tau_{23}+\tau_{13} \tau_{24}
$$


Then there is a commutative diagram

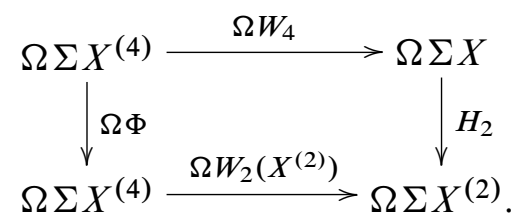

Proof The second Hopf invariant of the 4-fold Whitehead product

$$
\Omega \Sigma X^{(4)} \stackrel{\Omega W_{4}}{\longrightarrow} \Omega \Sigma X \stackrel{H_{2}}{\longrightarrow} \Omega \Sigma X^{(2)}
$$

restricted to $X^{(4)}$ has as its algebraic counterpart the coalgebra map

$$
V^{\otimes 4} \stackrel{\beta_{4}}{\longrightarrow} T(V) \stackrel{H_{2}}{\longrightarrow} T\left(V^{\otimes 2}\right) .
$$

Recall that

$$
\begin{aligned}
\beta_{4}\left(x_{1} x_{2} x_{3} x_{4}\right)= & x_{1} x_{2} x_{3} x_{4}-x_{2} x_{1} x_{3} x_{4}-x_{3} x_{1} x_{2} x_{4}+x_{3} x_{2} x_{1} x_{4} \\
& -x_{4} x_{1} x_{2} x_{3}+x_{4} x_{1} x_{2} x_{3}+x_{4} x_{3} x_{1} x_{2}-x_{4} x_{3} x_{2} x_{1} .
\end{aligned}
$$

According to the Cohen-Taylor combinatorial formulae [5] for the Hopf invariant

$$
H_{2}\left(a_{1} a_{2} a_{3} a_{4}\right)=a_{1} a_{2} a_{3} a_{4}+a_{1} a_{3} a_{2} a_{4}+a_{2} a_{3} a_{1} a_{4},
$$

the direct calculation gives

$$
H_{2} \circ \beta_{4}\left(x_{1} x_{2} x_{3} x_{4}\right)=\left[x_{1} x_{2}, x_{4} x_{3}\right]-\left[x_{2} x_{1}, x_{4} x_{3}\right]+\left[x_{4} x_{1}, x_{3} x_{2}\right]+\left[x_{3} x_{1}, x_{4} x_{2}\right] .
$$

By taking the geometrical realization of the above formulae, we obtain the map $S_{2}\left(X^{(2)}\right) \circ \Phi$, where $S_{2}\left(X^{(2)}\right)$ is the 2 -fold Samelson product. Notice that the Samelson product is the adjoint of the Whitehead product. Hence by taking the (unique) multiplicative extension, the assertion follows.

\section{Application: The rate of exponent growth for the Barratt Conjecture}

In this section we are concerned with an application of combinatorial method to the exponent problem in homotopy theory. Closely related to the exponent problem is the Barratt Conjecture stated as follows. If $f: \Sigma^{2} X \longrightarrow Y$ is of order $p^{r}$ in $\left[\Sigma^{2} X, Y\right]$, then

$$
p^{r+1} \operatorname{Im}\left(f_{*}: \pi_{*}\left(\Sigma^{2} X\right) \longrightarrow \pi_{*}(Y)\right)=0 .
$$

Algebraic ${ }^{6} \mathcal{G}$ Geometric Topology, Volume 6 (2006) 
In particular, if the identity map on $\Sigma^{2} X$ has order $p^{r}$ in $\left[\Sigma^{2} X, \Sigma^{2} X\right]$, then the exponent of $\Sigma^{2} X$ is $p^{r+1}$, that is,

$$
p^{r+1} \pi_{*}\left(\Sigma^{2} X\right)=0
$$

A stronger version of the Barratt Conjecture is concerned with the multiplicative exponent of $\Sigma^{2} X$ and can be stated as follows. If $f: \Sigma^{2} X \longrightarrow Y$ is of order $p^{r}$ in $\left[\Sigma^{2} X, Y\right]$, then

$$
\Omega^{2} f: \Omega^{2} \Sigma^{2} X \longrightarrow \Omega^{2} Y
$$

has an order bounded by $p^{r+1}$ in $\left[\Omega^{2} \Sigma^{2} X, \Omega^{2} Y\right]$.

An early result due to M G Barratt [1] gave a bound on the rate of the exponent growth for the Barratt Conjecture. The next theorem states the analogue rate of the exponent growth but for the stronger version of the Barratt Conjecture. In this section we assume that $R=\mathbb{Z} / p^{r}$.

Theorem 4.1 Let $X=\Sigma X^{\prime}$ be a suspension and let $f: X \longrightarrow \Omega Y$ be a map such that $p^{r}[f]=0$ in the group $[X, \Omega Y]$. Let $J(f): J(X) \longrightarrow \Omega Y$ be the canonical multiplicative extension of $f$. Then the following hold.

(1) The map

$$
\left.J(f)\right|_{J_{n}(X)}: J_{n}(X) \longrightarrow \Omega Y
$$

has order $p^{r+t}$ in $\left[J_{n}(X), \Omega Y\right]$ if $n<p^{t+1}$.

(2) The composite

$$
J_{p^{t+1}}(X) \stackrel{J(f)||_{p^{t+1}}(X)}{\longrightarrow} \Omega Y \stackrel{p^{r+t}}{\longrightarrow} \Omega Y
$$

is homotopic to the composite

$$
J_{p^{t+1}}(X) \stackrel{\text { pinch }}{\longrightarrow} X^{\left(p^{t+1}\right)} \stackrel{p^{r-1}\left(\sum_{\tau \in \Sigma_{p^{t+1}-1}} 1 \wedge \tau\right)}{\longrightarrow} X^{\left(p^{t+1}\right)} \stackrel{\widetilde{W}_{p^{t+1}}}{\longrightarrow} J(X) \stackrel{J(f)}{\longrightarrow} \Omega Y,
$$

where $p^{r+t}: \Omega Y \longrightarrow \Omega Y$ is the $p^{r+t}$-th power map, $\widetilde{W}_{n}$ is the $n$-fold Samelson product and

$1 \wedge \tau\left(x_{1} \wedge \cdots \wedge x_{p^{t+1}}\right)=x_{1} \wedge x_{\tau(2)} \wedge \cdots \wedge x_{\tau\left(p^{t+1}\right)}: X^{\left(p^{t+1}\right)} \longrightarrow X^{\left(p^{t+1}\right)}$

is the map which permutes positions.

(3) Let $g=J(f) \circ \widetilde{W}_{p^{t+1}} \circ\left(\sum_{\tau \in \Sigma_{p} t+1-1} 1 \wedge \tau\right) \circ p^{r-1}: X^{\left(p^{t+1}\right)} \longrightarrow \Omega Y$. Then $g$ is an equivariant map with respect to the symmetric group action, that is,

$$
g \circ \sigma \simeq g
$$

for any $\sigma \in \Sigma_{p^{t+1}}$. 
Remark The map

$$
p^{r-1}\left(\sum_{\tau \in \Sigma_{p^{t+1}-1}} 1 \wedge \tau\right): X^{\left(p^{t+1}\right)} \longrightarrow X^{\left(p^{t+1}\right)}
$$

is well-defined because $X$ is a suspension and so $\left[X^{\left(p^{t+1}\right)}, X^{\left(p^{t+1}\right)}\right]$ is an abelian group.

Proof Recall that the ground ring $R$ is $\mathbb{Z} / p^{r} \mathbb{Z}$. As $f: X \longrightarrow \Omega Y$ has order $p^{r}$, Corollary 2.15 implies that the homomorphism $e_{f}: \mathcal{H} \longrightarrow[J(X), \Omega Y]$ factors through the quotient group $\mathcal{H}^{\mathbb{Z} / q}$, where $q=p^{r}$. There is an induced homomorphism $e_{f}^{\mathbb{Z} / q}: \mathcal{H}^{\mathbb{Z} / q} \longrightarrow[J(X), \Omega Y]$. Further, recall that there is a group isomorphism $e: \mathcal{H}^{\mathbb{Z} / q} \longrightarrow \mathrm{Coalg}^{\mathbb{Z} / q}(T(-), T(-))$.

To show that the exponent of $J_{n}(f)$ is $p^{r+t}$ for $n<p^{t+1}$, consider first the counterpart of the $p^{r+t}$-th power map $p^{r+t}: J(X) \longrightarrow J(X)$ on the level of natural coalgebra transformations of the tensor algebra $T(-)$, that is, the $p^{r+t}$-th convolution power of the identity $\mathrm{Id}_{T}$

$$
\phi_{t}=\mathrm{Id}^{* p^{r+t}}: T \stackrel{\Psi_{p^{r+t}-1}}{\longrightarrow} T^{\otimes p^{r+t}} \stackrel{\mu}{\longrightarrow} T .
$$

Note that $\operatorname{Hom}_{R}(T, T)$ is an algebra under the convolution product with $\operatorname{Coalg}(T, T) \subseteq \operatorname{Hom}_{R}(T, T)$. The identity 1 in the ring $\operatorname{Hom}_{R}(T, T)$ is the composite

$$
\text { 1: } T \stackrel{\epsilon}{\longrightarrow} R \stackrel{v}{\longrightarrow} T \text {. }
$$

Let $\overline{\mathrm{Id}}=\operatorname{Id}_{T}-1$ in $\operatorname{Hom}_{R}(T, T)$ which is represented by the composite

$$
\overline{\mathrm{Id}}: T \longrightarrow I T \longrightarrow T .
$$

Then

$$
\begin{gathered}
\mathrm{Id}^{* p^{r+t}}=(1+\overline{\mathrm{Id}})^{p^{r+t}}=1+\sum_{k=1}^{p^{r+t}}\left(\begin{array}{c}
p^{r+t} \\
k
\end{array}\right) \overline{\mathrm{Id}}^{* k} \\
=1+\left(\begin{array}{c}
p^{r+t} \\
p^{t}
\end{array}\right) \overline{\mathrm{Id}}^{* p^{t}}+\sum_{k=p^{t}+1}^{p^{r+t}}\left(\begin{array}{c}
p^{r+t} \\
k
\end{array}\right) \overline{\mathrm{Id}}^{* k}
\end{gathered}
$$

in $\operatorname{Hom}_{R}(T, T)$ because $\left(\begin{array}{c}p^{r+t} \\ i\end{array}\right) \equiv 0 \bmod p^{r}$ for $1<i<p^{t}$. The $k$-th convolution power $\overline{\mathrm{Id}}^{* k}$ is represented by the composite

$$
T \stackrel{\Psi_{k-1}}{\longrightarrow} T^{\otimes k} \longrightarrow I T^{\otimes k} \stackrel{\mu}{\longrightarrow} T .
$$

Algebraic $8 \mathcal{G}$ Geometric Topology, Volume 6 (2006) 
Now the restriction map $\left.\overline{\mathrm{Id}}^{* k}\right|_{p^{t}}=0$ for $k>p^{t}$ because $I T^{\otimes k}$ is a summand of $T_{k}$ and a collection of $T_{j}$ 's with $j>k$. Moreover, $\left.\overline{\mathrm{Id}}^{* p^{t}}\right|_{p_{p^{t}}}$ is represented by the composite

$$
J_{p}(V) \longrightarrow T_{p^{t}}(V)=V^{\otimes p^{t}} \stackrel{\sum_{\sigma \in \Sigma_{p} t}}{\longrightarrow} V^{\otimes p^{t}} \longrightarrow T(V),
$$

where $\Sigma_{p^{t}}$ acts on $V^{\otimes p^{t}}$ by permuting positions. It follows that

$$
\overline{\mathrm{Id}}_{T}^{* p^{t}} \in \operatorname{ker}\left(\operatorname{Coalg}\left(J_{p^{t}}, T\right) \longrightarrow \operatorname{Coalg}\left(J_{p^{t}-1}, T\right)\right) \cong \operatorname{Lie}^{R}\left(p^{t}\right)
$$

represented by the element $\left(\begin{array}{c}p^{r+t} \\ p^{t}\end{array}\right) \sum_{\sigma \in \Sigma_{p} t} \sigma$. We need to rewrite this element in terms of Lie elements. Let $\bar{V}_{n}$ be the free $R$-module with a basis $\left\{x_{1}, \ldots, x_{n}\right\}$ and let $\gamma_{n}^{R}$ be the $R$-submodule of $\bar{V}^{\otimes n}$ generated by the homogenous elements $x_{\sigma(1)} x_{\sigma(2)} \cdots x_{\sigma(n)}$ for $\sigma \in S_{n}$. Let $\operatorname{Lie}^{R}(n)$ be the $R$-submodule of $\gamma_{n}^{R}$ generated by the $n$-fold commutators $\left[\left[x_{\sigma(1)}, x_{\sigma(2)}, \cdots x_{\sigma(n)}\right]\right.$ for $\sigma \in S_{n}$. Let

$$
\begin{aligned}
\operatorname{tr}_{n} & =\sum_{\sigma \in \Sigma_{n}} x_{\sigma(1)} x_{\sigma(2)} \cdots x_{\sigma(n)} \in \gamma_{n} \subseteq \bar{V}^{\otimes n} \text { and } \\
\overline{\operatorname{tr}}_{n} & =\sum_{\tau \in \Sigma_{n-1}}\left[\left[x_{1}, x_{\tau(2)}\right], x_{\tau(3)}, \cdots, x_{\tau(n)}\right] \in \operatorname{Lie}^{R}(n)
\end{aligned}
$$

be the sum of the standard basis for $\gamma_{n}$ and $\operatorname{Lie}^{R}(n)$, respectively. As $R=\mathbb{Z} / p^{r}$,

$$
\begin{gathered}
p^{r+t-1}\left(x_{1}+x_{2} \cdots+x_{p^{l}}\right)^{p^{l}}=p^{r+t-1} \operatorname{tr}_{p^{l}}+W \\
=p^{r+t-1}\left(x_{1}+v\right)^{p^{l}}=p^{r+t-1}\left[\left[x_{1}, v\right], v, \ldots v\right]+W^{\prime}=p^{r+t-1} \overline{\operatorname{tr}}_{p^{l}}+W
\end{gathered}
$$

where $v=x_{2}+x_{3}+\cdots x_{p^{l}}, W$ is a sum of the homogeneous terms in which one of the $x_{i}$ 's occurs at least twice and $W^{\prime}$ is a sum of the homogeneous terms in which the number of occurrences of $x_{1}$ is 0 or greater than 1 . Thus

$$
p^{r+t-1} \operatorname{tr}_{p^{l}}=p^{r+t-1} \overline{\operatorname{tr}}_{p^{l}}
$$

and so

$$
\operatorname{Id}_{T}^{* p^{r+t}}=\left(\begin{array}{c}
p^{r+t} \\
p^{t}
\end{array}\right) \overline{\operatorname{tr}}_{p^{t}}
$$

in $\operatorname{Lie}^{R}\left(p^{r}\right)$.

Being an element of the Cohen group $\overline{\operatorname{tr}}_{n}$ has a geometrical realization given by the composite

$$
\overline{\operatorname{tr}}: J_{n}(\Sigma X) \stackrel{\text { pinch }}{\longrightarrow}(\Sigma X)^{(n)} \stackrel{\sum_{\tau \in \Sigma_{n-1}} \mathrm{id}_{\Sigma X} \wedge \tau}{\longrightarrow}(\Sigma X)^{(n)} \stackrel{W_{n}}{\longrightarrow} \Omega \Sigma^{2} X \stackrel{\Omega f}{\longrightarrow} \Omega Y
$$


for $f: \Sigma^{2} X \longrightarrow Y$.

Now consider the case when $n=p^{t+1}$. Then

$$
\begin{aligned}
& e\left(\left(x_{1} \cdots x_{p^{t}}\right)^{p^{r+t}}\right)=1+\left(\begin{array}{c}
p^{r+t} \\
p^{t+1}
\end{array}\right) \sum_{\sigma \Sigma_{p^{t}}} y_{\sigma(1)} \cdots y_{\sigma\left(p^{t}\right)} \\
& =1+p^{r-1} q \sum_{\sigma \in \Sigma_{p^{t}}} y_{\sigma(1)} \cdots y_{\sigma(n)} .
\end{aligned}
$$

Cohen, Moore and Neisendorfer [3] proved that

$$
\sum_{\tau \in \Sigma_{p^{t}-1}}\left[\left[y_{1}, y_{\tau(2)}, \cdots, y_{\tau\left(p^{t}\right)}\right] \equiv \sum_{\sigma \in \Sigma_{p^{t}}} y_{\sigma(1)} \cdots y_{\sigma(n)} \quad(\bmod p)\right.
$$

where $\Sigma_{p^{t}-1}$ acts on $\left\{2, \cdots, p^{t}\right\}$. Thus

$$
p^{r-1} \sum_{\tau \in \Sigma_{p^{t}-1}}\left[\left[y_{1}, y_{\tau(2)}, \cdots, y_{\tau\left(p^{t}\right)}\right]=p^{r-1} \sum_{\sigma \in \Sigma_{p^{t}}} y_{\sigma(1)} \cdots y_{\sigma(n)} .\right.
$$

Assertions (2) and (3) follow.

For $X$ a $p$-local suspension space, we now consider the special map

$$
\phi_{n}=\sum_{\sigma \in \Sigma_{n}} \sigma: X^{(n)} \longrightarrow X^{(n)},
$$

where $\Sigma_{n}$ acts on $X$ by permuting positions.

Lemma 4.2 Let $\alpha \in \mathbb{Z}_{(p)}\left(\Sigma_{n}\right)$, such that $\alpha=\sum_{\sigma \in \Sigma_{n}} k_{\sigma} \sigma$. Then

$$
\phi_{n} \circ \alpha=\left(\sum_{\sigma \in \Sigma_{n}} k_{\sigma}\right) \phi_{n} .
$$

Proof The statement follows from the observation that $\phi_{n} \circ \sigma=\phi_{n}$ for any permutation $\sigma \in \Sigma_{n}$.

Lemma 4.3 Let $f=\sum_{\sigma \in \Sigma_{n}} k_{\sigma} \sigma \in \mathbb{Z}\left(\Sigma_{n}\right)$, where $k_{\sigma} \in \mathbb{Z}$, such that the sum of the coefficients

$$
\chi(f)=\sum_{\sigma \in \Sigma_{n}} k_{\sigma} \not \equiv 0 \quad(\bmod p) .
$$

Let $X$ be a $p$-torsion suspension of finite type. Then there exists a map

$$
\phi_{n}(f): \operatorname{hocolim}_{f} X^{(n)} \longrightarrow \operatorname{hocolim}_{f} X^{(n)}
$$

Algebraic $8 \mathcal{G}$ Geometric Topology, Volume 6 (2006) 
such that the diagram

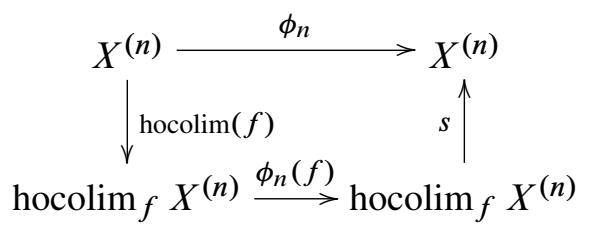

commutes up to homotopy, where $s$ : hocolim $_{f} X^{(n)} \longrightarrow X^{(n)}$ is a map such that $\operatorname{hocolim}(f) \circ s$ : $\operatorname{hocolim}_{f} X^{(n)} \longrightarrow \operatorname{hocolim}_{f} X^{(n)}$ is the identity map.

Proof We may assume that $\chi(f)=1$. Notice that

$$
\phi_{n}=f^{q} \circ \phi_{n} \circ f^{r}
$$

for any $q, r \geq 1$, where $f^{m}$ is the $m$-fold composition $f \circ f \circ \cdots \circ f$. The assertion follows.

Assume that $X$ is a suspension. Let $M_{n}(X)$ be the smallest functorial retract of $X^{(n)}$ that contains the bottom cell in the sense of papers $[9 ; 4]$.

Proposition 4.4 Let $X$ be a suspension. Then the trace map

$$
\phi_{n}=\sum_{\sigma \in \Sigma_{n}} \sigma: X^{(n)} \longrightarrow X^{(n)}
$$

factors through the smallest functorial retract $M_{n}(X)$ of $X^{(n)}$.

Proof By construction of $M_{n}(X)$, there exists an idempotent $e_{n}$ in the group algebra $\mathbb{Z}_{(p)}\left(\Sigma_{n}\right)$ such that $e_{n}=\sum_{\sigma \in \Sigma_{n}} k_{\sigma} \sigma$ with $\sum_{\sigma \in \Sigma_{n}} k_{\sigma}=1$ and the induced geometric map $e_{n}: X^{(n)} \longrightarrow X^{(n)}$ has the homotopy colimit

$$
M_{n}(X)=\operatorname{hocolim}_{e_{n}} X^{(n)} .
$$

Now the result follows from Lemma 4.2.

Corollary 4.5 Let $X$ be a suspension. Then the composite

$$
J_{p^{t+1}}(X) \stackrel{\left.J(f)\right|_{p_{p^{t+1}}(X)} ^{\longrightarrow}}{\longrightarrow} \Omega Y \stackrel{p^{r+t}}{\longrightarrow} \Omega Y
$$

is homotopic to the composite

$$
J_{p^{t+1}}(X) \stackrel{\text { pinch }}{\longrightarrow} X^{\left(p^{t+1}\right)} \longrightarrow X \wedge M_{p^{t+1}-1}(X) \longrightarrow X^{\left(p^{t+1}\right)} \stackrel{W_{p^{t+1}}}{\longrightarrow} J(X) \stackrel{J(f)}{\longrightarrow} \Omega Y .
$$


The homology of $M_{n}(X)$ is unknown for a general $X$. The determination of the homology of $M_{n}(X)$ is equivalent to the fundamental problem in the modular representation theory of the symmetric group $\Sigma_{n}$, that of determining the decomposition of the group ring into indecomposable summands.

In the case of two cell complexes the homology of $M(X)$ is well understood. Let $S_{n}(V)$ denote the $n$-th homogeneous component of the symmetric algebra on $V$.

Theorem 4.6 [4, Corollary 1.5] Let $X$ be a two-cell suspension. Then

$$
\tilde{H}_{*}\left(M_{n}(X)\right) \cong S_{n}\left(\tilde{H}_{*}(X)\right)
$$

provided that $n=c p^{r}-1$ for some $1 \leq c \leq p-1$.

Theorem 4.6 gives a special meaning to Corollary 4.5, claiming that the first obstruction to the Barratt Conjecture for a two cell complex $X$ factors through the very small space $X \wedge M_{p^{t+1}-1}(X)$.

Let us consider a particular example in which $p=2$. Let $X$ be a suspension. We write $[q]: X \longrightarrow X$ for the co-H $q$-th power map for any integer $q$.

Proposition 4.7 Let $X=P^{n}(2)$ be the $n$-dimensional mod 2 Moore space with $n \geq 3$. Then the composite

$$
X^{(3)} \stackrel{[2]}{\longrightarrow} X^{(3)} \stackrel{\sum_{\sigma \in \Sigma_{3}} \sigma}{\longrightarrow} X^{(3)}
$$

is null homotopic and therefore by the Theorem 1.3 (2), the power map

$$
\left.8\right|_{J_{4}\left(P^{n}(2)\right)}: J_{4}\left(P^{n}(2)\right) \longrightarrow J\left(P^{n}(2)\right) \simeq \Omega\left(P^{n+1}(2)\right)
$$

is null homotopic.

Proof Let (123) be the 3-cycle in $\Sigma_{3}$ and let $f=(123)+(123)^{2}+(123)^{3}$. Then one can check that $\operatorname{hocolim}_{f} X^{(3)} \simeq \mathbb{C} P^{2} \wedge P^{3 n-4}(2)$, as in [11]. By Lemma 4.3, there exists a map $\phi_{3}(f): \mathbb{C} P^{2} \wedge P^{3 n-4}(2) \longrightarrow \mathbb{C} P^{2} \wedge P^{3 n-4}(2)$ such that the map $\sum_{\sigma \in \Sigma_{3}} \sigma: X^{(3)} \longrightarrow X^{(3)}$ is homotopic to the composite

$$
X^{(3)} \longrightarrow \mathbb{C} P^{2} \wedge P^{3 n-4}(2) \stackrel{\phi_{3}(f)}{\longrightarrow} \mathbb{C} P^{2} \wedge P^{3 n-4}(2) \longrightarrow X^{(3)} .
$$

Notice that

$$
\phi_{3}(f)_{*}: H_{3 n-3}\left(\mathbb{C} P^{2} \wedge P^{3 n-4}(2) ; \mathbb{Z} / 2\right) \longrightarrow H_{3 n-3}\left(\mathbb{C} P^{2} \wedge P^{3 n-4}(2) ; \mathbb{Z} / 2\right)
$$

is zero. Thus $\phi_{3}(f)$ restricted to the bottom cell is null homotopic. Notice that $\pi_{3 n-2}\left(\mathbb{C} P^{2} \wedge P^{3 n-4}(2)\right)=0$. Thus $\phi_{3}(f)$ restricted to the $(3 n-2)-$ skeleton of 
the 4-cell complex $\mathbb{C} P^{2} \wedge P^{3 n-4}(2)$ is null homotopic. Thus there exists a map $\bar{\phi}: P^{3 n}(2) \longrightarrow \mathbb{C} P^{2} \wedge P^{3 n-4}(2)$ such that $\phi_{3}(f)$ is homotopic to the composite

$$
\mathbb{C} P^{2} \wedge P^{3 n-4}(2) \stackrel{q}{\longrightarrow} P^{3 n}(2) \stackrel{\bar{\phi}}{\longrightarrow} \mathbb{C} P^{2} \wedge P^{3 n-4}(2),
$$

where $q$ is the pinch map. Notice that the map [2]: $P^{3 n}(2) \longrightarrow P^{3 n}(2)$ is homotopic to the composite

$$
P^{3 n}(2) \stackrel{\text { pinch }}{\longrightarrow} S^{3 n} \stackrel{\eta}{\longrightarrow} S^{3 n-1} \longrightarrow P^{3 n}(2) .
$$

Thus the pinch map $q: \mathbb{C} P^{2} \wedge P^{3 n-4}(2) \longrightarrow P^{3 n}(2)$, which is a suspension map, is of exponent 2 in the group $\left[\mathbb{C} P^{2} \wedge P^{3 n-4}(2), P^{3 n}(2)\right]$. The assertion follows.

\section{References}

[1] M G Barratt, Spaces of finite characteristic, Quart. J. Math. Oxford Ser. (2) 11 (1960) 124-136 MR0120647

[2] F R Cohen, On combinatorial group theory in homotopy theory, preprint

[3] F R Cohen, J C Moore, J A Neisendorfer, Torsion in homotopy groups, Ann. of Math. (2) 109 (1979) 121-168 MR519355

[4] F R Cohen, P Selick, J Wu, Natural decompositions of self-smashes of 2-cell complexes, preprint

[5] F R Cohen, L R Taylor, Homology of function spaces, Math. Z. 198 (1988) 299-316 MR946606

[6] J Grbić, J Wu, Natural transformations of tensor algebras and representations of combinatorial groups, Algebr. Geom. Topol. 6 (2006) 2189-2228

[7] J A Neisendorfer, The exponent of a Moore space, from: "Algebraic topology and algebraic $K$-theory (Princeton, N.J., 1983)", Ann. of Math. Stud. 113, Princeton Univ. Press (1987) 35-71 MR921472

[8] P Selick, J Wu, On natural coalgebra decompositions of tensor algebras and loop suspensions, Mem. Amer. Math. Soc. 148 (2000) MR1706247

[9] P Selick, J Wu, On functorial decompositions of self-smash products, Manuscripta Math. 111 (2003) 435-457 MR2002820

[10] S Theriault, Exponents of mod- $2^{r}$ Moore spaces, to appear in Topology

[11] J Wu, On maps from loop suspensions to loop spaces and the shuffle relations on the Cohen groups, to appear in Memoirs AMS

[12] J Wu, On combinatorial calculations for the James-Hopf maps, Topology 37 (1998) 1011-1023 MR1650426 
Department of Mathematical Sciences, University of Aberdeen Meston Building, Aberdeen AB24 3UE, UK

Department of Mathematics, National University of Singapore 2 Science Drive 2, Singapore

jelena@maths.abdn.ac.uk, matwuj@nus.edu.sg

Received: 23 October 2006 